\title{
Deep Sequencing of Chicken microRNAs
}

\author{
Joan Burnside*1, Ming Ouyang3, Amy Anderson1, Erin Bernberg1, Cheng Lu², \\ Blake C Meyers $^{2}$, Pamela J Green ${ }^{2}$, Milos Markis ${ }^{1}$, Grace Isaacs ${ }^{1}$, \\ Emily Huang ${ }^{1}$ and Robin W Morgan ${ }^{1}$
}

\begin{abstract}
Address: ${ }^{1}$ Department of Animal and Food Sciences, Delaware Biotechnology Institute, University of Delaware, Newark, Delaware, 19711, USA, ${ }^{2}$ Department of Plant and Soil Sciences, Delaware Biotechnology Institute, University of Delaware, Newark, Delaware, 19711, USA and ${ }^{3}$ University of Louisville, Department of Computer Engineering \& Computer Science Louisville, Kentucky, 40292, USA

Email: Joan Burnside* - joan@udel.edu; Ming Ouyang - ming.ouyang@louisville.edu; Amy Anderson - amya@udel.edu; Erin Bernberg - elb@udel.edu; Cheng Lu - lu@dbi.udel.edu; Blake C Meyers - meyers@dbi.udel.edu; Pamela J Green - green@dbi.udel.edu; Milos Markis - markis@udel.edu; Grace Isaacs - gkisaacs@udel.edu; Emily Huang - ehuang@udel.edu; Robin W Morgan - morgan@udel.edu

* Corresponding author
\end{abstract}

Published: 22 April 2008

BMC Genomics 2008, 9:185 doi:10.1/86/147|-2164-9-185
Received: 10 November 2007

Accepted: 22 April 2008

This article is available from: http://www.biomedcentral.com//47/-2/64/9/185

(C) 2008 Burnside et al; licensee BioMed Central Ltd.

This is an Open Access article distributed under the terms of the Creative Commons Attribution License (http://creativecommons.org/licenses/by/2.0), which permits unrestricted use, distribution, and reproduction in any medium, provided the original work is properly cited.

\begin{abstract}
Background: The use of new, deep sequencing technologies has greatly accelerated microRNA discovery. We have applied this approach to the identification of chicken microRNAs and to the comparison of microRNAs in chicken embryo fibroblasts (CEF) infected with Marek's disease virus (MDV) to those present in uninfected CEF.

Results: We obtained 125,463 high quality reads that showed an exact match to the chicken genome. The majority of the reads corresponded to previously annotated chicken microRNAs; however, the sequences of many potential novel microsRNAs were obtained. A comparison of the reads obtained in MDV-infected and uninfected CEF indicates that infection does not significantly perturb the expression profile of microRNAs. Frequently sequenced microRNAs include miR-22I/ 222, which are thought to play a role in growth and proliferation. A number of microRNAs (e.g., let-7, miR-199a-I, 26a) are expressed at lower levels in MDV-induced tumors, highlighting the potential importance of this class of molecules in tumorigenesis.

Conclusion: Deep sequencing technology is highly suited for small RNA discovery. This approach is independent of comparative sequence analysis, which has been the primary method used to identify chicken microRNAs. Our results have confirmed the expression of many microRNAs identified by sequence similarity and identified a pool of candidate novel microRNAs.
\end{abstract}

\section{Background}

MicroRNAs are small (about $22 \mathrm{nt}$ ) RNAs that play important regulatory roles by targeting mRNAs for degradation or translational repression. MicroRNAs were first identified in Caenorhabditis elegans [1] but high evolutionary conservation eventually led to the identification of microRNAs in other species. This, coupled with conventional sequencing of small RNA libraries, has greatly expanded the list of known microRNAs. The most recent release of the microRNA database, miRBase 10.0 [2], contains over 5000 microRNA gene loci in a wide variety of animal, plant and viral genomes. 
Conventional sequencing favors identification of highly expressed species, and comparative genomics will not identify nonconserved microRNAs. In order to enhance discovery of small RNA species, massively parallel signature sequencing (MPSS) was used to identify small RNAs in Arabidopsis thaliana [3], and the results showed that the diversity of small RNAs exceeded previous estimates. More recently, newer deep sequencing technologies have been used to profile microRNAs in Arabidopsis DICER and RDR2 mutants $[4,5]$, and others have applied this technology to various samples including human and chimpanzee brain [6] and Chlamydomonas reinhardtii [7]. These approaches have the advantage that they not only provide sequence of low abundance species, but also provide quantitative data since the frequency of sequencing reads reflects the abundance of microRNAs in the population.

We previously reported on the use of deep sequencing technologies for identification of microRNAs encoded by Marek's disease virus (MDV), an economically important pathogenic herpesvirus of chickens $[8,9]$. In an extension of the pilot study, we sequenced additional reads from both MDV-infected chicken embryo fibroblasts (CEF) and uninfected CEF and now report on the identification of potential novel host microRNAs. In addition, the sequence of several new MDV-encoded microRNAs were discovered by deeper sequencing.

\section{Results}

\section{Small RNA libraries}

We obtained 256,221 reads from two small RNA libraries prepared from uninfected CEF or CEF infected with MDV. As shown in Table 1, a total of 171,783 reads contained both adapters used in creating the library, and 125,463 of these high quality reads showed an exact match to the chicken genome. A total of 1,036 reads from the MDVinfected CEF library matched the MDV genome. The presence of other small RNAs (ribosomal fragments, tRNA, snRNA, mtRNA) was relatively small (less than 3\%).

The majority ( $86 \%$ ) of the small RNAs match to known or predicted chicken microRNAs (Additional File 1). Of the 149 distinct Gallus gallus (gga) entries in miRbase, we found 101 distinct species expressed in CEF. There were
93 matches from the MDV-infected CEF library and 87 matches from the uninfected CEF library. The infected cells showed slightly more complexity in microRNA diversity, which may be in part due to the larger number of reads obtained from the infected CEF library which increases the chances of revealing low abundance microRNAs. There were 12 microRNAs in the infected cells that were not found in the uninfected CEFs and 9 microRNAs found in the uninfected CEFs that were not found in the infected cells. An additional eleven chicken homologs of known microRNAs were identified (Additional File 1). The size distribution of reads was not significantly different in the two libraries, and the majority of the reads had lengths of 21-25 nt (Figure 1).

\section{microRNA profiling by analysis of read counts}

The number of reads obtained should reflect the relative abundance and expression levels of the microRNAs. After scaling for total number of reads obtained for each library, the majority of microRNAs were found at similar levels in the two libraries. A few microRNAs (listed in Table 2) showed a greater than two-fold difference in the number of reads between the infected and uninfected CEF libraries. We found miR-29b and miR-196 at higher levels in the MDV-infected cells, and three of the let7 microRNAs were found at lower levels in the MDV-infected CEF compared to the uninfected CEF. Northern blot analysis did not detect these differences, but this could be because of the low read numbers or because of cross hybridization with microRNAs with similar sequences (miR-29a, let7 family).

The most frequently sequenced ( $>500$ reads) microRNAs are found at remarkably similar levels in the two libraries (Table 3). Consistent with findings in our pilot study [9], the highest number of reads was obtained for gga-miR222 and 221. These are clustered on chromosome 1 (114216027-114219024), and in the chicken there are two copies of miR-222 in the cluster, which could account for the higher number of miR-222 reads. We also see high levels of gga-miR-125b/148a/21 and 103.

Table I: Distribution of small RNAs from uninfected CEF and CEF infected with MDV

\begin{tabular}{lcc}
\hline & MDV infected CEF & uninfected CEF \\
\hline High quality/both adapters & 107,728 & 64,055 \\
Exact match to chicken genome & 79,074 & 46,389 \\
Match to known miRNAs & 67,982 & 40,173 \\
Match to other chicken smalls' & 3,249 & 1,487 \\
Match to MDV & 1036 & - \\
Other potential smalls & 7,761 & 4,666 \\
\hline
\end{tabular}

ItRNA, rRNA, mtRNA, snRNA 


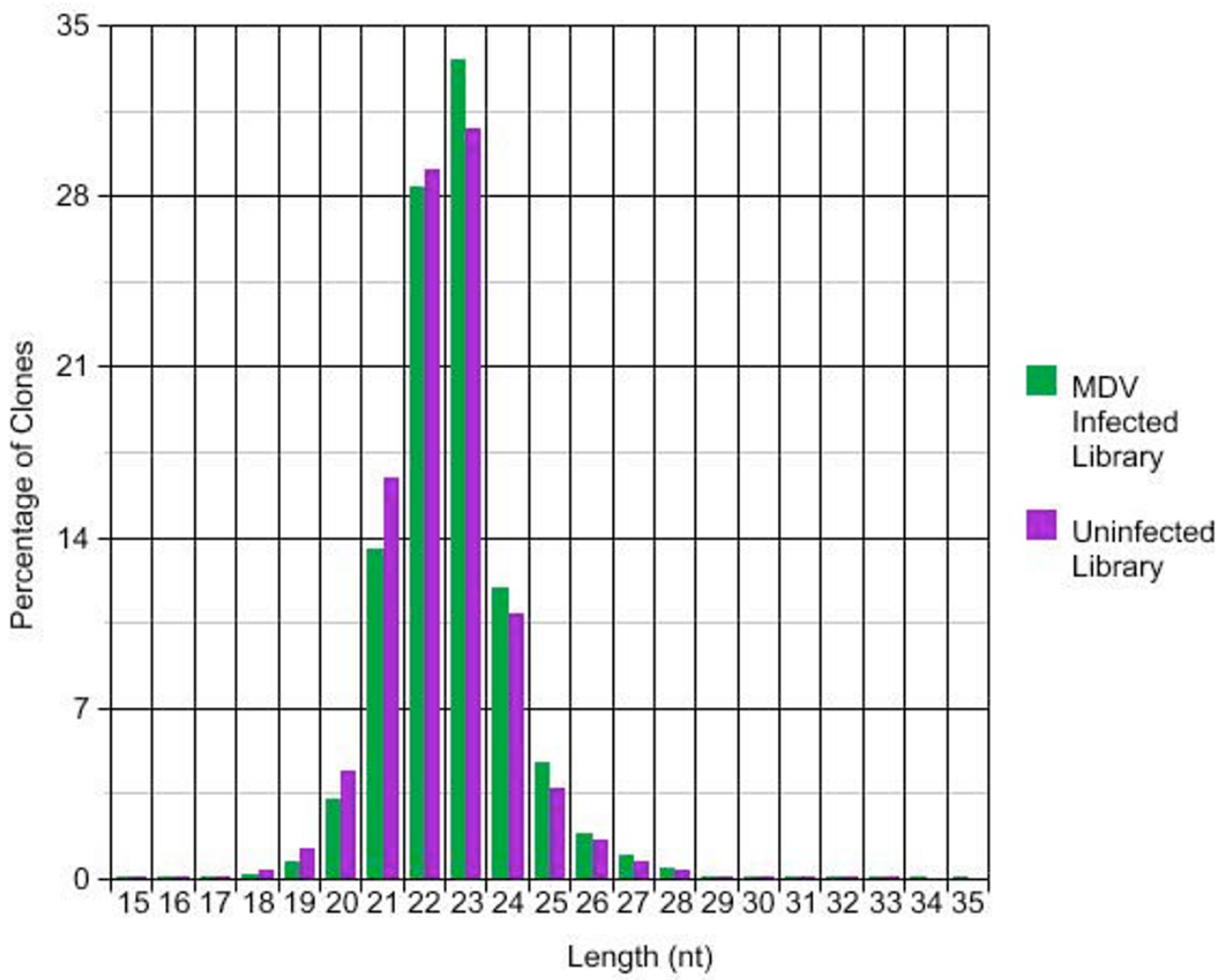

Figure I

Size distribution of small RNAs.

\section{Viral microRNAs}

We previously identified ten MDV-encoded microRNAs in a pilot sequencing project of the MDV-infected CEF library [9]. The deep sequencing revealed an additional seven microRNAs and ' $*$ ' strands (Table 4). Four of the new microRNAs map to the previously identified LAT cluster (mdv1-miR- $6^{*}, 8^{*}, 10$, and $\left.10^{*}\right)$, two are in the cluster upstream of the meq gene (mdv1-miR-11 and $5^{*}$ ), one is downstream of the meq gene (mdv1-miR-12), and one is antisense to the coding region of the ribonucleotide reductase gene (mdv1-miR-M9). (A preliminary discussion of some of these microRNAs was reviewed in [8]).

Table 2: Relative abundance of differentially expressed microRNAs

\begin{tabular}{|c|c|c|c|c|c|}
\hline microRNA name & Length & Sequence & $\begin{array}{l}\text { \# Reads in MDV } \\
\text { infected CEF }\end{array}$ & $\begin{array}{c}\text { \# Reads in } \\
\text { uninfected CEF }\end{array}$ & $\begin{array}{c}\text { Ratio Infected/Uninfected } \\
\text { (Normalized) }\end{array}$ \\
\hline gga-miR-29b & 23 & TAGCACCATTTGAAATCAGTGTT & 64 & 2 & 18.8 \\
\hline gga-miR-196 & 21 & TAGGTAGTTTCATGTTGTTGG & 23 & I & 13.5 \\
\hline gga-miR-133a & 22 & TTGGTCCCCTTCAACCAGCTGT & 15 & 2 & 4.4 \\
\hline gga-miR-I0b & 22 & TACCCTGTAGAACCGAATTTGT & 70 & 15 & 2.7 \\
\hline gga-miR-30d & 22 & TGTAAACATCCCCGACTGGAAG & 23 & 6 & 2.3 \\
\hline gga-let-7f & 22 & TGAGGTAGTAGATTGTATAGTT & 338 & 454 & 0.4 \\
\hline gga-let-7b & 22 & TGAGGTAGTAGGTTGTGTGGTT & 74 & 105 & 0.4 \\
\hline gga-miR-I30a & 22 & CAGTGCAATATTAAAAGGGCAT & 18 & 28 & 0.4 \\
\hline gga-let-7a & 22 & TGAGGTAGTAGGTTGTATAGTT & 383 & 603 & 0.4 \\
\hline gga-miR-la & 21 & TGGAATGTAAAGAAGTATGTA & 7 & 12 & 0.3 \\
\hline
\end{tabular}

Data shown are microRNAs with more than 15 reads in one library and 2-fold difference in read count, after scaling for total number of reads matching the chicken genome in each library. 
Table 3: Most frequently sequenced microRNAs in CEFs

\begin{tabular}{lccc}
\hline microRNA name & \# Reads in MDV infected CEF & \# Reads in uninfected CEF & Ratio Infected/Uninfected (normalized) \\
\hline gga-miR-222a & 10361 & 4945 & 1.2 \\
gga-miR-22I & 6708 & 4112 & 1.0 \\
gga-miR-125b & 3689 & 2093 & 1.0 \\
gga-miR-148a & 3583 & 2062 & 1.0 \\
gga-miR-2I & 2929 & 1488 & 1.2 \\
gga-miR-103 & 1297 & 841 & 0.9 \\
gga-miR-17-5p & 826 & 564 & 0.9 \\
gga-miR-20a & 666 & 414 & 0.9 \\
gga-miR-27b & 650 & 400 & 1.0 \\
gga-miR-20b & 619 & 378 & 1.0 \\
gga-miR-199 & 529 & 301 & 1.0 \\
gga-miR-26a & 522 & 334 & 0.9 \\
gga-miR-218 & 499 & 370 & 0.8 \\
\hline
\end{tabular}

Data shown are microRNAs with more than 15 reads in one library and 2-fold difference in read count, after scaling for total number of reads matching the chicken genome in each library.

\section{Potential novel microRNAs}

About $10 \%$ of the reads matched the chicken genome but not other known small RNAs and were considered candidates for novel microRNAs. The presence of hairpin structures containing these reads was evaluated using RNAfold [10], and those present in hairpins were further filtered according to established criteria [11]. First, the candidate microRNA is entirely within the arm of the hairpin that has the lowest free energy among all sliding windows containing the candidate microRNA. Second, at least sixteen of 22 nucleotides of the candidate microRNA must match the other arm of the hairpin. Third, the hairpin should not contain any large (> $5 \mathrm{nt}$ ) internal loops or bulges. Matches to repeats or regions of low complexity were eliminated. Additional File 2 lists 63 candidate novel
microRNAs passing these criteria. Uracil, which is preferentially found in the first position of known chicken microRNAs, is also first in $48 \%$ of the candidate novels. None contain a seed sequence that is identical to already established microRNA families. Three of the candidates (ID \#26/27,38/39 and 50/51) are found in the same stem loop, making it likely that they are mature and ' $*$ ' strands of premicroRNAs. One (ID \#10) is clustered $96 \mathrm{nt}$ upstream of gga-miR-7-2, and one (ID\#31) is immediately upstream of gga-let-7a-2.

Curiously, one of the potential novels (\#ID14) is found within the highly evolutionarily conserved coding region of DCGR8 (DiGeorge syndrome critical region gene 8),

Table 4: MDV encoded microRNAs

\begin{tabular}{|c|c|c|c|}
\hline Name and Sequence (5' -> 3') & Length & \# Reads & MDV/IRL Position \\
\hline mdvl-miR-MI: TGCTTGTTCACTGTGCGGCAI & 20 & 304 & 136873 \\
\hline mdv1-miR-M2: GTTGTATTCTGCCCGGTAGTCCGI & 23 & 191 & |3423| \\
\hline mdvl-miR-M2*: CGGACTGCCGCAGAATAGCTTI & 21 & 16 & 134270 \\
\hline mdvI-miR-M3: ATGAAAATGTGAAACCTCTCCCGCI & 24 & 13 & 134080 \\
\hline mdvl-miR-M4: TTAATGCTGTATCGGAACCCTTC' & 23 & 206 & 134368 \\
\hline mdvl-miR-M4*: AATGGTTCTGACAGCATGACC' & 21 & 6 & 134405 \\
\hline mdvl-miR-M5: TGTGTATCGTGGTCGTCTACTGTI & 23 & 62 & 133647 \\
\hline mdvl-miR-M5*CGTATGCGATCACATTGACACG & 22 & 12 & 133609 \\
\hline mdvI-miR-M6: GAGATCCCTGCGAAATGACAGTI & 22 & 87 & 142370 \\
\hline mdvl-miR-M6*: TGTTGTTCCGTAGTGTTCTCG & 21 & 39 & 142335 \\
\hline mdvI-miR-M7: TCGAGATCTCTACGAGATTACAGI & 23 & 15 & 142547 \\
\hline mdvl-miR-M8: GTGACCTCTACGGAACAATAGTI & 22 & 50 & 142258 \\
\hline mdvl-miR-M8* TATTGTTCTGTGGTTGGTTTCG & 23 & II & 142216 \\
\hline mdvI-miR-M9: TGTTGATCCGTAGATAGGCGATGGC & 25 & 5 & 96961 \\
\hline mdvI-miR-MI0: GCGTTGTCTCGTAGAGGTCCAGI & 22 & 4 & 142627 \\
\hline mdvl-miR-MI0*: TCGAAATCTCTACGAGATAACAGTT & 25 & 2 & 142669 \\
\hline mdv I-miR-M I I: TTGCATAATACGGAGGGTTCTG & 22 & 3 & 133925 \\
\hline mdv1-miR-MI2: TGCTACAGTCGTGAGCAGATCAA & 23 & 10 & $13658 \mid$ \\
\hline
\end{tabular}


which interacts with Drosha in the processing of primicroRNAs [12].

The expression of several of these candidate microRNAs was evaluated by northern blot analysis of different tissues (Figure 2). All hybridize to species the size of mature microRNAs. Some of the novel microRNAs are expressed ubiquitously (ID\#26, 39, 51), while others show more selective expression (ID\#46,61). These microRNAs show no sequence similarity to any known microRNAs, with the exception of \#46, which is similar, but not identical to dre-miR-730 (21/22) and gga-miR-460 (19/22). The microRNAs analyzed by Northern blots were selected based on presence of star strand in sequencing data, presence in a cluster, or some level of sequence conservation with other species. Other candidate microRNAs in the list have not been evaluated.

\section{Expression of known host microRNAs in MDV-induced tumors}

There is a large and growing literature on microRNA expression in tumors, and both up- and down-regulation have been observed, with microRNA expression patterns reflecting the developmental history and lineage of neoplasms. We compared expression in MDV-induced tumors versus normal spleen tissue for selected host microRNAs that were either differentially expressed based on the deep sequencing or that were interesting based on the literature. Figure 3 shows that the expression of ggamiR-let 7, 199a-1, 26a, 181a, and -16 were all expressed at lower levels in tumors, compared to normal spleens, using either gga-miR-221 or U6 as a loading control; ggamiR-221 is slightly lower in tumors.

\section{Discussion}

Our deep sequencing approach to microRNA discovery in the chicken confirms the expression of 112 known microRNAs and identifies a pool of 63 candidate novel microRNAs. The majority of the known chicken microRNAs have been identified by sequence comparison with microRNAs from other species [13], and the expression of many has been confirmed by analysis of EST data and in situ hybridization $[14,15]$. Cloning from small RNA libraries also validates the expression of microRNAs. A recent study of chicken microRNA cloning [16] used conventional technology to confirm expression of 25 of the known chicken microRNAs and identified one possible novel microRNA. Our study adds to the confirmation of expression of predicted microRNAs, and greatly expands the list of potential novel microRNAs in the chicken.

A large majority $(86 \%)$ of the reads from chicken CEF small RNA libraries matched known microRNAs. Similar numbers were obtained in deep sequencing of human brain [6], where $80 \%$ of the reads matched known human
microRNAs. Thus, it could be argued that we are approaching saturation of microRNA discovery. However, it is possible that only highly and ubiquitously expressed microRNAs have been found, and less abundant or tissuespecific microRNAs may still be revealed by deep sequencing of different tissues. This is clearly the case in plants, where a large set of small RNAs have been discovered by deep sequencing [3]. In an analysis of the human 'colorectal microRNAome', SAGE was used as a deep sequencing approach, and matches to 200 microRNAs in miRbase were found, as well as 100 previously unrecognized microRNA* strands and 133 candidate novels [17]. MPSS analysis of mouse embryo small RNA discovered over 60 potential novel microRNAs, some of which are rodent specific [11]. We have identified a pool of 63 candidate novel chicken microRNAs and have confirmed expression of several candidates by northern blot analysis. Some are expressed in a tissue-specific manner, while others are more ubiquitously expressed. Further analysis of these and other candidates with respect to temporal and tissue expression will be a first step to understanding function. Overall, the deep sequencing approach to microRNA discovery suggests that a significant number of novel microRNAs remain to be discovered and characterized.

In addition to identifying novel chicken microRNAs, deep sequencing of MDV infected CEF has revealed previously uncharacterized MDV-encoded microRNAs, bringing the total of MDV microRNAs to 18. Other herpesviruses encode microRNAs, and these are thought to play a role in immune evasion, apoptosis and cell cycle control $[18,19]$. MDV causes a well- characterized, virally induced T cell lymphoma of chickens and represents an excellent model system for analyzing the function of viral microRNAs in the pathogenesis of cancer. Many recent studies implicate microRNAs as either tumor suppressors or oncogenes [20], and host encoded microRNAs can act in cis (on viral target genes) or trans (on host encoded genes) to affect phenotypic changes [21]. Moreover, virally infected cells are stressed, and it has been proposed that microRNAs play an important role in the stress response [22]. A comparison of the reads of MDV-infected CEF versus uninfected CEF indicates that the majority of the microRNAs are expressed at similar levels. However, CEF are used to propagate virus, and viral infection occurs in a very small percentage of cells, thus making it difficult to observe changes when analyzing whole cultures. In addition, CEF are not the in vivo target of the virus, and we might expect a more critical layer of regulation in T cells. A small set of microRNAs appeared to be differentially expressed in infected vs. uninfected cells, but this was not confirmed by northern blot analysis. This lack of concordance between the two techniques is not uncommon when expression levels are very low, or when cross-hybridization with similar species can confound the results. Additionally, in our 


\begin{tabular}{lllr} 
A. & & & \\
ID\# & Sequence & Chromosome & \multicolumn{1}{c}{ Position } \\
50 & TTCGATGCTTGTATGCTACTCC & chr7 & 1330109 \\
25 & TCACACCAGAGTAACTGGGATCGATC & chr2 & 141373945 \\
38 & CGTAACTCGCTGCTGTGAGAGGC & chr3 & 78710269 \\
45 & CACAGCGCATGCAATGTGGACATT & chr4 & 2687452 \\
58 & TCCTTAACTCATGCCGCTGTGCT & chrZ & 34596495
\end{tabular}

B.
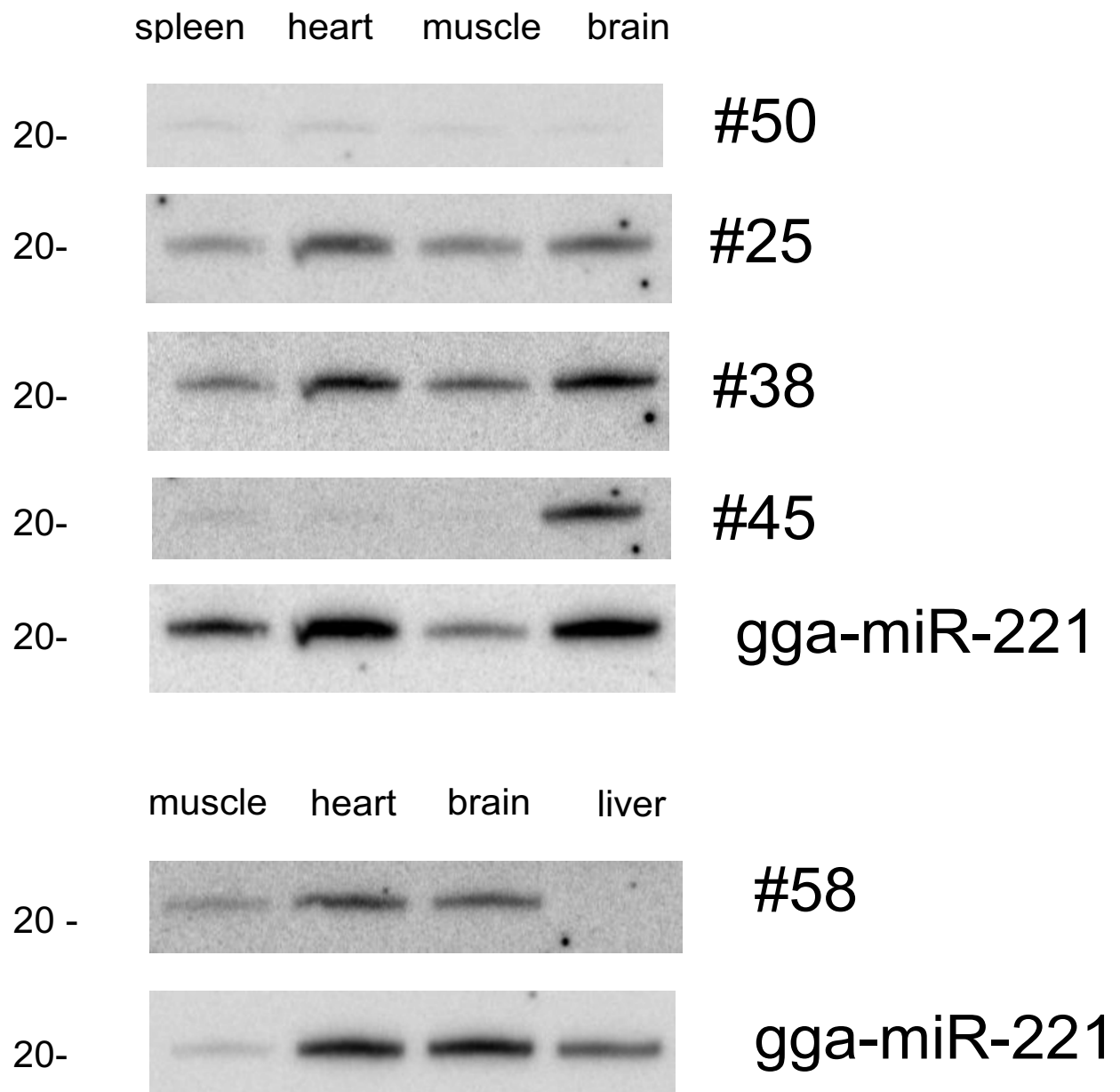

\section{Figure 2}

Sequence and expression of novel chicken microRNAs. A. Sequence and chromosomal location of selected novel microRNAs (location is based on May 2006 build). B. Northern blot analysis of individual microRNAs shows relative expression in different tissues. Blots were hybridized to gga-miR-22I to verify presence of microRNAs in each lane. 


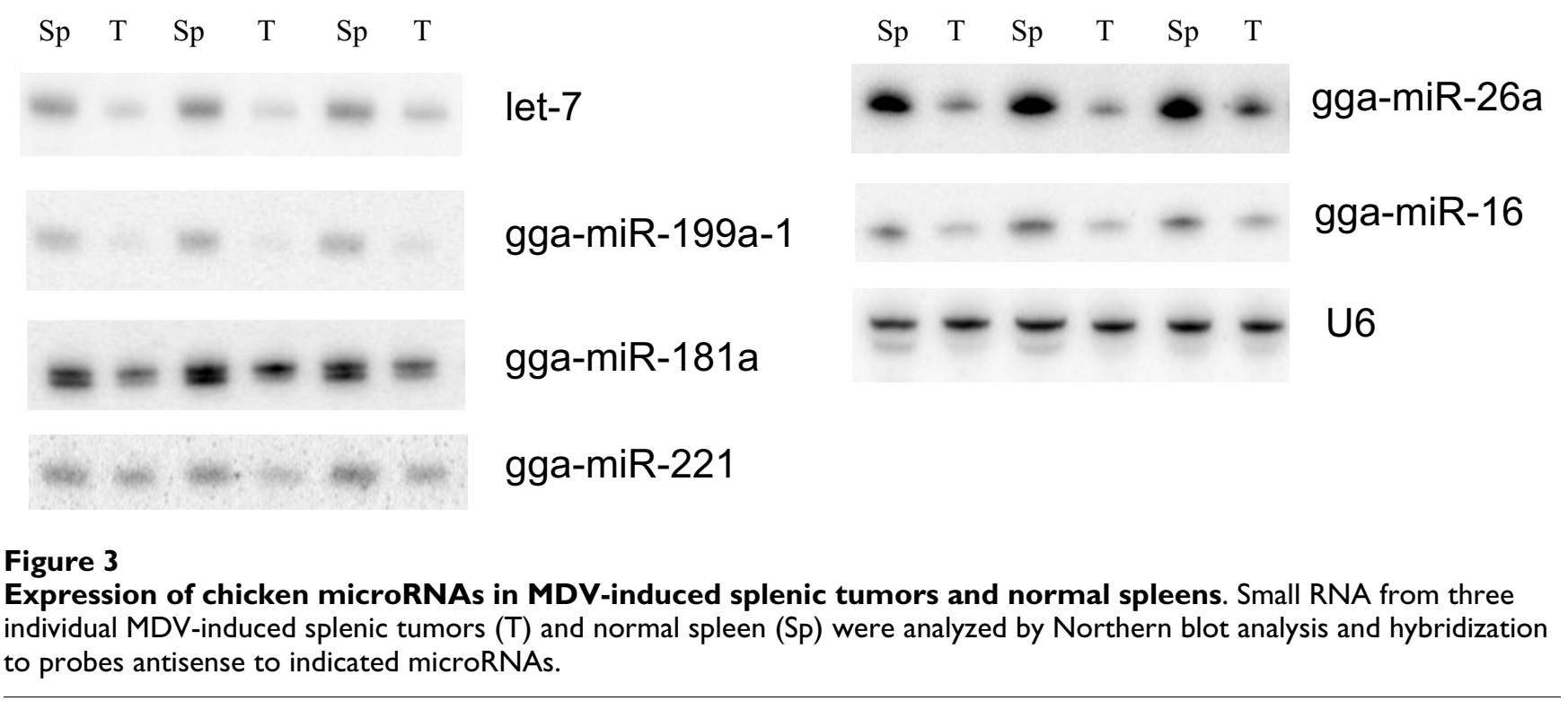

system, MDV infects only a small percentage of the cells and infections vary considerably from culture to culture. This biological noise could also hamper the ability to reproduce differences noted in the sequencing data set.

The two most frequently sequenced microRNAs were ggamiR-222 and -221, which also share sequence identity in the seed region. These are located within a $3000 \mathrm{nt}$ region of Chromosome 1, where there are two copies of gga-miR222 followed by one copy of gga-miR-221. In human, miR-221 and -222 are coordinately expressed from a single primary transcript [23]. We see about 1.7 -fold higher abundance of ggg-miR-222 compared to gga-miR-221, consistent with their sharing a transcript that is highly expressed in CEF. Computational analysis has predicted that p27Kip1 protein, a key inhibitory regulator of the cell cycle [24], is a potential target for this cluster. Down regulation of $\mathrm{p} 27 \mathrm{Kip} 1$ by miR-221/222 promotes growth and proliferation of cancer cells, and could play a similar role in dividing CEF [24]. miR-125b and 21 were also abundant in our libraries. miR-125b is critical for the proliferation of some human cell lines [25], and mir-21 is thought to function as an oncogene by decreasing apoptosis [26]. The high levels in rapidly dividing CEF could play a permissive role in the cell cycle in CEF.

Our northern analysis of MDV-induced tumors shows several host microRNAs that were noticeably less abundant in MDV-induced tumor tissue compared to normal spleen, consistent with previous reports of a general down-regulation of microRNAs in tumors [27]. Among those down regulated, let7 was particularly interesting. The let7 microRNA is known to down-regulate Hmga2 [28], which is a small, non-histone, chromatin-associated protein that is believed to influence chromatin remodeling [29]. Hmga2 is expressed robustly in undifferentiated proliferating cells, and its expression during embryogenesis and in a variety of benign and malignant tumors has been characterized [30]. Down-regulation of let7 should lead to increased expression of Hmga-2, and such a scenario would be consistent with the cell proliferation that characterizes tumors. miR-16 is considered a tumor suppressor [31], which acts by targeting BCL-2, and repressed expression is consistent with tumorigenesis. MiR-181s were down-regulated in gliobastoma compared to normal brain controls [32], and miR-199a was down-regulated in hepatocelluar carcinoma [33]. Thus, it is likely that in MDV-induced tumors, as in other tumors, many microRNAs act collectively to facilitate cellular transformation and proliferation. More information on the perturbations of host microRNAs will come from a deep sequencing analysis of microRNAs in tumors.

\section{Conclusion}

Understanding the biological function of microRNAs first requires identification of all microRNAs within a genome. Here we have described the application of deep sequencing technology for the identification of many candidate novel chicken microRNAs from a single tissue source. The application of this technology to other tissues will no doubt lead to the identification of other novel microRNAs, which will improve the annotation of the chicken genome and further our understanding of this important class of regulatory molecules. 


\section{Methods}

Cloning, sequencing and analysis of chicken microRNAs

Secondary CEF, prepared by routine techniques, were infected with the RB1B strain of MDV as described previously [9]. Protocols developed previously in the Green lab were used to construct the small RNA libraries [3]. cDNA inserts were amplified by PCR, and amplicons were sequenced by 454 Life Sciences [34]. Sequence data were filtered for adapter sequences, clustered (allowing a 4base overhang or mismatch at either end), and insert sequence was analyzed by comparing to the chicken and MDV genomes, chicken ncRNA (Ensembl 12/06) and to the microRNA database [2] using Perl string matching. The remaining sequences were analyzed using RNAfold [10] to identify the loop structure of minimum free energy containing the microRNAs. This list was further curated to eliminate highly repetitive sequences [35].

\section{Northern blot analysis of microRNAs}

RNA was fractionated using PEG or through use of the FlashPage system (Ambion) as described previously [9]. The low molecular weight fractions were electrophoresed on a $15 \%$ denaturing polyacrylamide gel, electroblotted to charged nylon, and hybridized to ${ }^{32}$ P-labeled antisense primers complementary to the microRNAs. Hybridization to an antisense primer for gga-miR-221 or U6 was used as a loading control. A 10-bp DNA ladder was used to approximate size.

\section{Authors' contributions}

JB and RM designed and coordinated the study and wrote the manuscript; $\mathrm{MO}$ performed the analysis of the sequencing data; AA, EB and GI prepared samples for sequencing and for northern blots; CL prepared the libraries; PG and BM assisted in design, library construction and sequence analysis.

\section{Additional material}

Additional file 1
Expression of known microRNAs in CEF.
Click here for file
[http://www.biomedcentral.com/content/supplementary/1471-
2164-9-185-S1.xls]
Additional file 2
Candidate novel microRNAs.
Click here for file
[http://www.biomedcentral.com/content/supplementary/1471-
2164-9-185-S2.xls]

\section{Acknowledgements}

Funding for this work was provided by the USDA NRI. We would like to thank Evgeny.Glazov (CSRIO) for stimulating discussions and data sharing.

\section{References}

I. Lee RC, Feinbaum RL, Ambros V: The C. elegans heterochronic gene lin- 4 encodes small RNAs with antisense complementarity to lin-14. Cell 1993, 75:843-854.

2. [http://microrna.sanger.ac.uk/sequences/index.shtml].

3. Lu C, Tej SS, Luo S, Haudenschild CD, Meyers BC, Green PJ: Elucidation of the small RNA component of the transcriptome. Science 2005, 309: 1567-1569.

4. Henderson IR, Zhang X, Lu C, Johnson L, Meyers BC, Green PJ, jacobsen SE: Dissecting Arabidopsis thaliana DICER function in small RNA processing, gene silencing and DNA methylation patterning. Nature Genetics 2006, 38:721-725.

5. Lu C, Kulkarni K, Souret FF, MuthuValliappan R, Tej SS, Poethig RS, Henderson IR, Jacobsen SE, Wang W, Green PJ, et al.: MicroRNAs and other small RNAs enriched in the Arabidopsis RNAdependent RNA polymerase-2 mutant. Genome Res 2006, I 6( I 0): I276-1288.

6. Berezikov E, Thuemmler F, van Laake L, Kondova I, Bontrop R, Cuppen E, Plasterk R: Diversity of microRNAs in human and chimpanzee brain. Nature Genetics 2006, 38:1375-I377.

7. Ruby JG, Jan C, Player C, Axtell MJ, Lee W, Nusbaum C, Ge H, Bartel DP: Large-Scale Sequencing Reveals 2 IU-RNAs and Additional MicroRNAs and Endogenous siRNAs in C. elegans. Cell 2006, I 27: I 193-1207.

8. Burnside J, Morgan RW: Genomics and Marek's disease virus. Cytogenetic and Genome Research 2007, I I 7:376-387.

9. Burnside J, Bernberg E, Anderson A, Lu C, Meyers BC, Green PJ, Jain $\mathrm{N}$, Isaacs $\mathrm{G}$, Morgan RW: Marek's disease virus microRNAs map to meq and LAT. J Virology 2006, 80:8778-8786.

10. [http://www.tbi.univie.ac.at/ ivo/RNA/]

II. Mineno J, Okamoto S, Ando T, Sato M, Chono H, Izu H, Takayama M, Asada $K$, Mirochnitchenko $O$, Inouye $M$, et al.: The expression profile of microRNAs in mouse embryos. Nucl Acids Res 2006, 34(6): | 765-|77|.

12. Landthaler M, Yalcin A, Tuschl T: The human DiGeorge syndrome critical region gene 8 and its $D$. melanogaster homolog are required for miRNA biogenesis. Current Biology 2004, 14:2162-2167.

13. International CGSC: Sequence and comparative analysis of the chicken genome provide unique perspectives on vertebrate evolution. Nature 2004, 432:695-716.

14. Hubbard SJ, Grafham DV, Beattie KJ, Overton IM, McLaren SR, Croning MDR, Boardman PE, Bonfield JK, Burnside J, Davies RM, et al.: Transcriptome analysis for the chicken based on 19,626 finished cDNA sequences and 485,337 expressed sequence tags. Genome Res 2004, I 5:174-183.

15. Darnell DK, Kaur S, Stanislaw S, Konieczka JK, Yatskievyeh TA, Antin PB: MicroRNA expression during chick embryo development. Developmental Dynamics 2006, 235:3156-3165.

16. Xu H, Wang X, Du Z, Li N: Identification of microRNAs from different tissues of chicken embryo and adult chicken. FEBS Letters 2006, 580:3610-3616.

17. Cummins JM, He Y, Leary RJ, Pagliarini R, Diaz LA Jr, Sjoblom T, Barad O, Bentwich Z, Szafranska AE, Labourier E, et al:: The colorectal microRNAome. Proceedings of the National Academy of Sciences 2006, I 03(I0):3687-3692.

18. Sullivan CS, Ganem D: MicroRNAs and viral infection. Molecular Cell 2005, 20:3-7.

19. Cullen BR: Viruses and microRNAs. Nature Genetics 2006, 38:525-530.

20. Dalmay T, Edwards D: MicroRNAs and the hallmarks of cancer. Oncogene 2006, 25(6 I 70-6 I 75):6170-6I75.

21. Pfeffer S, Voinnet O: Viruses, microRNAs and cancer. Oncogene 2006, 25:6211-6219.

22. Leung AKL, Sharp PA: microRNAs: A safeguard against turmoil? Cell 2007, I 30:58I-585.

23. Poliseno L, Tuccoli A, Mariani L, Evangelista M, Citti L, Woods K, Mercatanti A, Hammond S, Rainaldi G: MicroRNAs modulate the angiogenic properties of HUVECs. Blood 2006, I08(9):3068-307I.

24. Galardi S, Mercatelli N, Giorda E, Massalini S, Frajese GV, Ciafre SA Farace MG: miR-22I and miR-222 Expression Affects the Proliferation Potential of Human Prostate Carcinoma Cell Lines by Targeting p27Kip I. J Biol Chem 2007, 282(32):237l6-23724.

25. Lee YS, Kim HK, Chung S, Kim K-S, Dutta A: Depletion of human micro-RNA miR-I25b reveals that It Is critical for theprolif- 
eration of differentiated cells but not for the down-regulation of putative targets during differentiation. J Biol Chem 2005, 280:16635-1664I.

26. Si M-L, Zhu S, Wu H, Lu Z, Wu F, Mo Y-Y: miR-2I-mediated tumor growth. Oncogene 2007, 26:2799-2803.

27. Lu J, Getz G, Miska EA, Alvarez-Saavedra E, Lamb J, Peck D, SweetCordero A, Ebert BL, Mak RH, Ferrando AA, et al.: MicroRNA expression profiles classify human cancers. Nature 2005, 435:834-843.

28. Mayr C, Hemann MT, Bartel DP: Disrupting the pairing between let-7 and Hmga2 enhances oncogenic transformation. Science 2007, 3 I 5(58 I 8): 1576-1579.

29. Sgarra R, Rustighi A, Tessari MA, Di Bernardo J, Altamura S, Fusco A, Manfioletti G, Giancotti V: Nuclear phosphoproteins HMGA and their relationship with chromatin structure and cancer. FEBS Letters 2004, 574: I-8.

30. Fedele M, Battista S, Manfioletti G, Croce CM, Giancotti V, Fusco A: Role of the high mobility group A proteins in human lipomas. Carcinogenesis 200I, 22(10): 1583-1591.

31. Cimmino A, Calin GA, Fabbri M, lorio MV, Ferracin M, Shimizu M, Wojcik SE, Aqeilan RI, Zupo S, Dono M, et al.: miR-I5 and miR-I6 induce apoptosis by targeting BCL2. Proceedings of the National Academy of Sciences 2005, I 22(39): I 3944- 13949.

32. Ciafre SA, Galardi S, Mangiola A, Ferracin M, Liu C-G, Sabatino G, Negrini M, Maira G, Croce CM, Farace MG: Extensive modulation of a set of microRNAs in primary glioblastoma. Biochemical and Biophysical Research Communications 2005, 334: I35I-1358.

33. Murakami Y, Yasuda T, Saigo K, Urashima T, Toyoda H, Okanoue T, Shimotohno K: Comprehensive analysis of microRNA expression patterns in hepatocellular carcinoma and non-tumorous tissues. Oncogene 2006, 25:2537-2545.

34. Margulies M, Egholm M, Altman WE, Attiya S, Bader JS, Bemben LA, Berka J, Braverman MS, Chen YJ, Chen Z, et al.: Genome sequencing in microfabricated high-density picolitre reactors. Nature 2005, 437(7057):376-380.

35. [http://www.repeatmasker.org/].

\section{Publish with Bio Med Central and every scientist can read your work free of charge}

"BioMed Central will be the most significant development for disseminating the results of biomedical research in our lifetime. "

Sir Paul Nurse, Cancer Research UK

Your research papers will be:

- available free of charge to the entire biomedical community

- peer reviewed and published immediately upon acceptance

- cited in PubMed and archived on PubMed Central

- yours - you keep the copyright

Submit your manuscript here:

http://www.biomedcentral.com/info/publishing_adv.asp
Biomedcentral 